\title{
Novel Molecular Diagnostic Platform for Tropical Infectious Diseases
}

\author{
Yasuyoshi Mori, Norihiro Tomita, \\ Hidetoshi Kanda and Tsugunori Notomi \\ Eiken Chemical Co., Ltd. \\ Japan
}

\section{Introduction}

Infectious disease is one of the most concerning health issues worldwide. To provide patients with effective medical treatment and prevent the spread of diseases and emergence of drug-resistant strains, quick and reliable diagnostic techniques are in high demand. However, lack of accessibility to such diagnostic systems has resulted in the deterioration of the situation in most developing countries, especially in sub-Saharan tropical countries (Rodrigues et al., 2010). Diagnostics using molecular technologies have emerged as a promising methodology because of their remarkable high sensitivity, and therefore, they have been applied as diagnostic tools for detecting various kinds of pathogens in clinical settings in developed countries. However, resources essential for molecular assays, such as bio-safety cabinets, a stable supply of electricity, and well-experienced technicians, are scarce in most of the peripheral laboratories in developing countries. In this chapter, we would like to describe a recently developed novel diagnostic platform and discuss its application for realizing molecular diagnostics for infectious diseases within resourcelimited settings.

Molecular diagnostics comprise the following 3 steps: sample preparation, amplification, and detection. To develop a molecular diagnostic platform with the desired simplicity and performance, it is necessary to introduce element technologies for all the 3 steps, which are less complicated and can be used in peripheral laboratories with limited resources. Of the abovementioned 3 steps, amplification of target DNA/RNA is the most important. Therefore, the loop-mediated isothermal amplification (LAMP) method involving the calcein detection method has been applied to the platform as a key technology. LAMP, using the calcein method, enables recognition of small quantities of DNA/RNA of pathogens present in clinical specimens by means of the fluorescence emitted from the LAMP solutions after amplification.

The next important step is sample processing, for which we have developed a simple and easy-to-use technology, namely, procedure for ultra rapid extraction (PURE). The combination of both these technologies can be considered a novel platform for molecular diagnostics, which can be applied to resource-limited settings. The fundamental characteristics of these element technologies and application of the novel platform to diagnostics for evaluation of certain tropical diseases are discussed below. 


\section{Steps involved in molecular diagnostics}

\subsection{Amplification - LAMP}

Since the publication of the first report regarding LAMP in 2000 (Notomi et al., 2000), LAMP has been used to detect different kinds of pathogens (Mori \& Notomi, 2009), including viruses (Kubo et al., 2010), bacteria (Iwamoto et al., 2003), and protozoa (Spencer et al., 2010), and thus far, approximately 500 reports have been published regarding the application of LAMP. Because the LAMP method is simple and quick, it has been considered one of the most ideal nucleic acid amplification methods, which can be applied as an easy-to-use and cost-effective genetic test system (Parida et al., 2008).

\subsubsection{Mechanism of LAMP}

Although the reaction mechanism appears complicated, LAMP is simple to perform-it involves mixing primers (designed as depicted in figure 1-A), DNA polymerase with stranddisplacement activity, and dNTPs, in a buffer containing magnesium ions, and maintaining the mixture at a constant temperature of $60-67{ }^{\circ} \mathrm{C}$ for $15-60$ minutes. If template DNA molecules are present in the sample solution, large quantities of DNA with the target sequence (amplicon) are produced after incubation.

Figures 1-B and $\mathrm{C}$ show the schematic representation of the mechanism of LAMP. First, the forward inner primer (FIP) anneals to the template DNA at the F2c sequence and the extension reaction occurs by the enzymatic activity of Bst polymerase. Because Bst polymerase exhibits strand displacement activity, the product obtained from FIP is displaced by the other extension reaction associated with the F3 primer. Subsequently, the extension reaction occurs from the backward inner primer (BIP) on the product of the FIP, and not on the template DNA with a B2C sequence; the product obtained is also displaced by DNA synthesis associated with the B3 primer. These reactions result in a product with a dumbbell-like structure as shown in figure 1-B. The formation of the dumbbell-like product is essential for LAMP to establish isothermal amplification because the loop structures are always single stranded and can be annealed by FIP or BIP. Thus, formation of the loop structure can lead to the elimination of the denaturing step, which is otherwise essential in PCR for obtaining single-stranded DNA.

After the formation of the dumbbell-like structure, a cyclic reaction is spontaneously established between the dumbbell-like structure and its complementary product, as shown in figure 1-C. Furthermore, in the course of the cyclic reaction, elongated products with various copies of the target sequence are also produced.

The basic characteristics of the LAMP method are summarized below:

1. The whole amplification reaction occurs continuously under isothermal conditions, thus eliminating the need to use a thermal cycler, which is commonly used for PCR.

2. Because LAMP primers recognize 6 distinct regions, the specificity of LAMP is much higher than that of the other commonly used amplification techniques.

3. Amplification can be performed using an RNA template only by the addition of reverse transcriptase to the reaction (one-step RT-LAMP).

4. The LAMP reaction can be accelerated by using additional primers, called "loop primers," which are designed between F1c/B1c and F2c/B2c (Nagamine et al., 2002). 


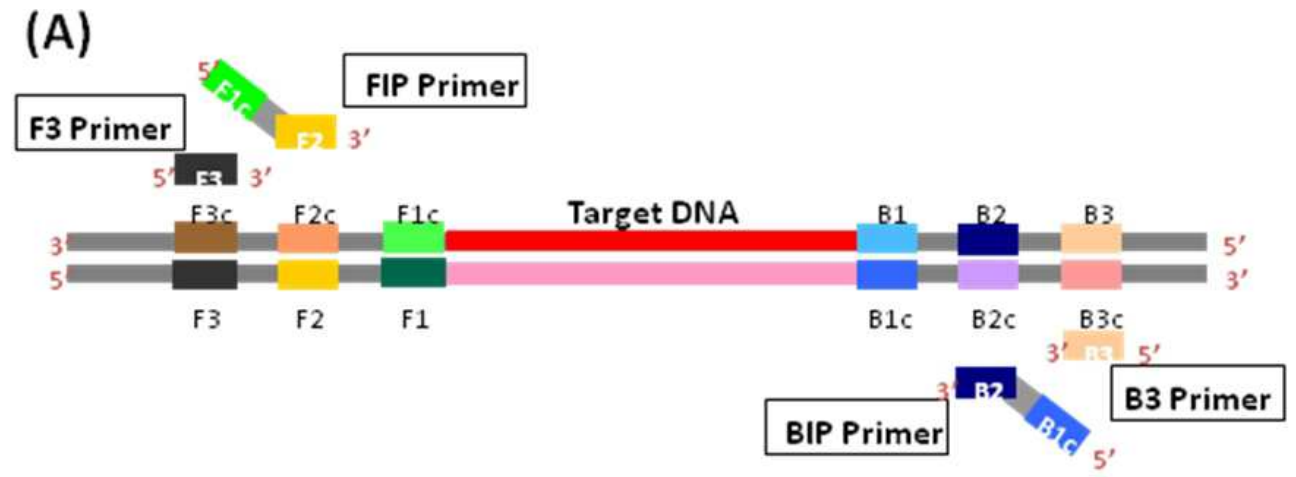

(B)
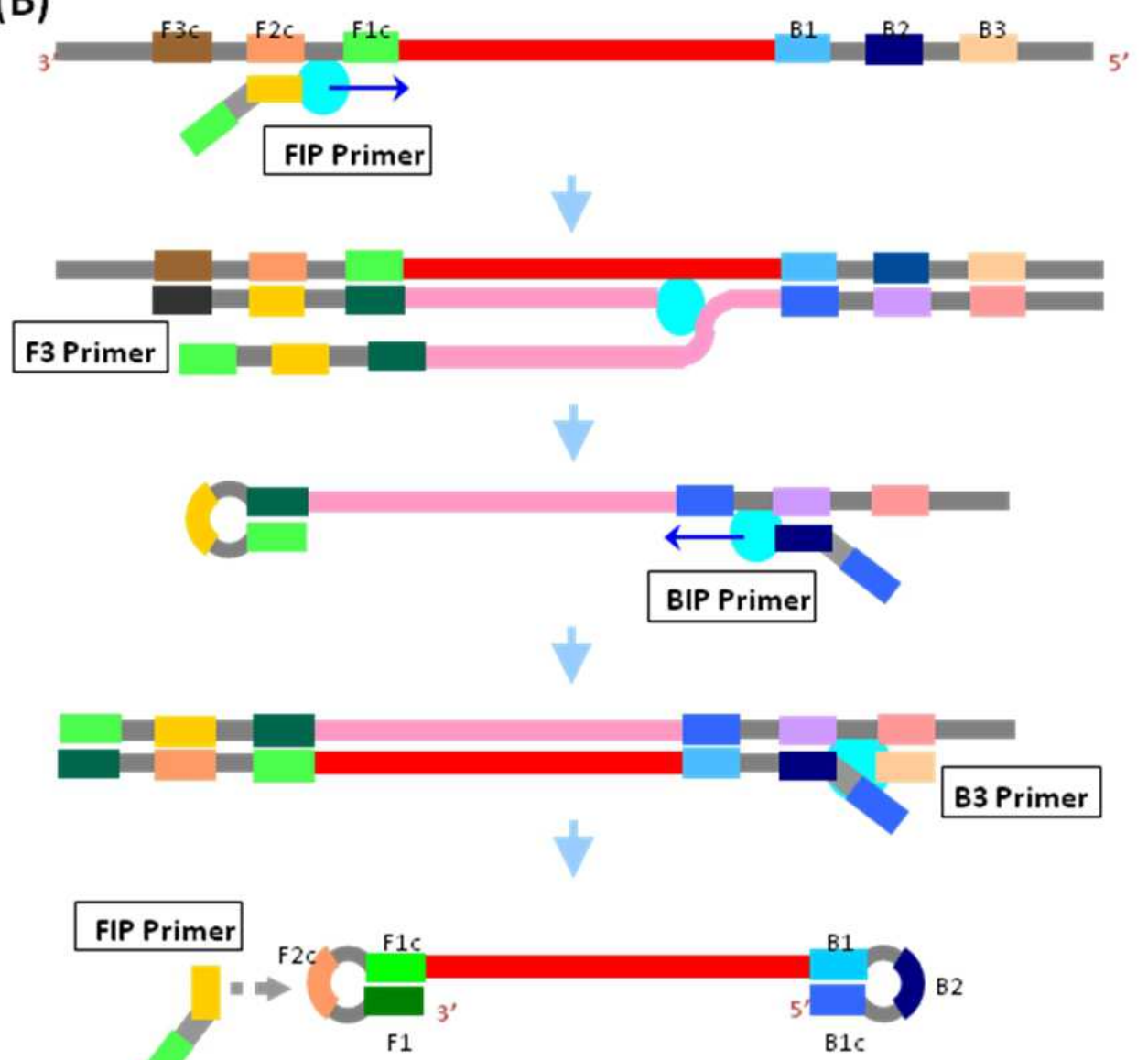


\section{(C)}

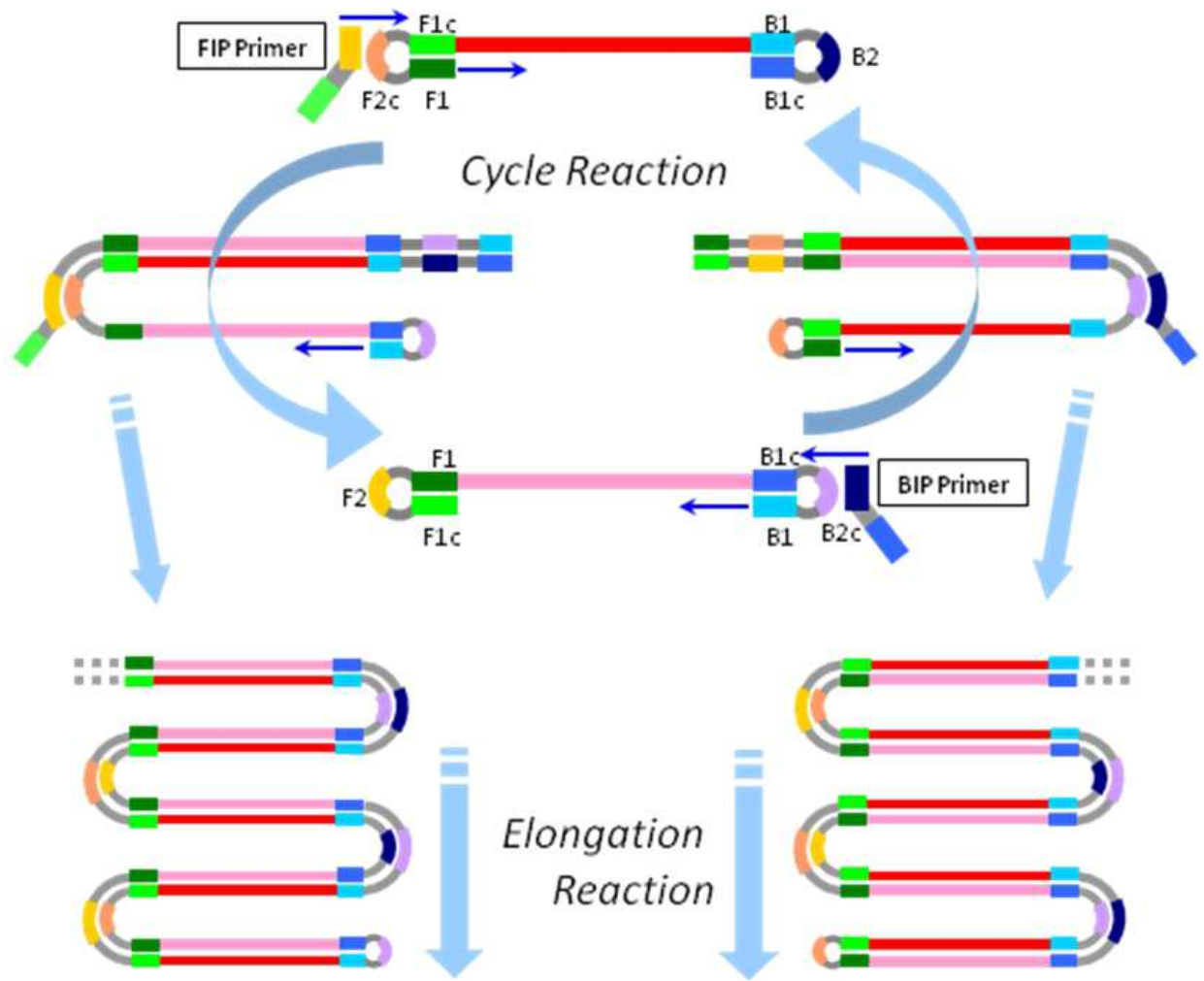

Fig. 1. Schematic representation of the mechanism of the LAMP assay

A) Design of the LAMP primers

B) Formation of a dumbbell-like structure

C) Cyclic and elongation reactions

\subsubsection{Strategies that make LAMP simple and cost-effective}

Conventional LAMP reagents are supplied in liquid form, and they have to be stored below $-20{ }^{\circ} \mathrm{C}$, similar to most PCR reagents. However, because of the lack of a freezer and cold chain transportation system in most of the peripheral laboratories in developing countries, it is essential to formulate LAMP reagents, which can be stably preserved at ambient temperatures (Jorgensen et al., 2006; Aziah et al., 2007). The newly formulated LAMP reagents are dried down into the lid of the reaction tubes, thus obtaining preservation stability at ambient temperatures for more than 12 months. The dried LAMP reagents can be reconstituted quite easily by shaking the tubes after the addition of the purified DNA solution. Because the LAMP reagent for each reaction is deposited on the individual tubes in advance, there is no longer a need to prepare and dispense master-mix solutions to the reaction tubes. Thus, liquid handling using micropipette, one of the most skillful steps, becomes unnecessary in the course of the assay. Moreover, this can contribute to reduced risk of carryover contamination during the assay. 


\subsection{Detection - Calcein method}

The results of the LAMP assay can be detected visually by observing the strength of the green fluorescence emitted after the reaction. Figure 2 represents the mechanism of the calcein method (Tomita et al., 2008). Before LAMP amplification, the metalochrome indicator "calcein" is quenched by the effect of a manganese ion. After the LAMP reaction, pyrophosphate ions (PPi) are produced as a by-product of polymerase reaction; PPi subsequently forms a manganese pyrophosphate complex, causing the removal of the manganese ion from calcein, because the PPi are a stronger base than calcein. Next, free calcein combines with a magnesium ion to produce bright fluorescence. This technology enables the detection of LAMP reactions without the use of fluorescence detectors, which are usually expensive and difficult to manage in resource-limited settings. Other technologies for visual detection using LAMP have also been reported (Tao et al., 2011; Goto et al., 2009; Mori et al., 2006).
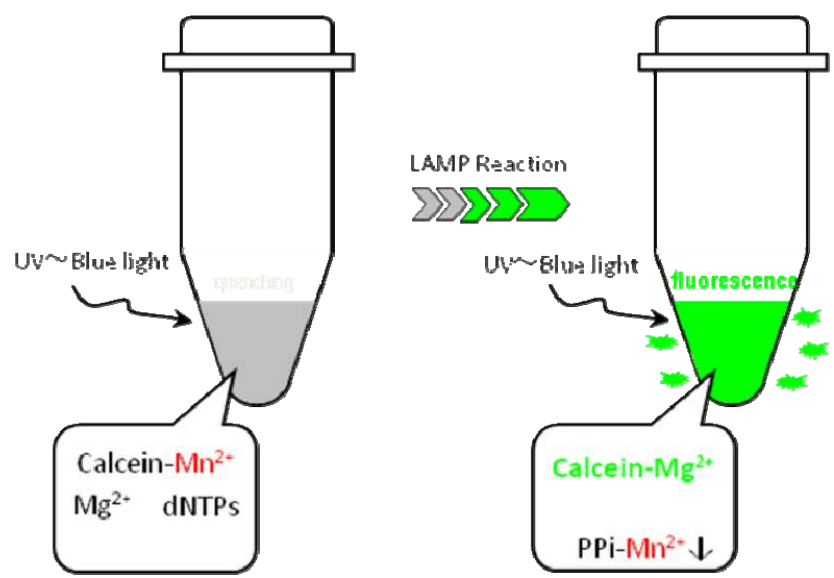

Fig. 2. Mechanism involved using calcein

\subsection{Sample preparation - PURE}

The sample processing method is the next important step in molecular diagnostics. Silicabased methods are well known and have been applied to a wide variety of samples, including blood and tissue (Bendall, 2002). However, these methods are unsuitable for resource-limited facilities due to the cumbersome procedures involved, including washing with organic solvents using high-speed centrifugation. Therefore, we have developed a simple and swift sample processing method named PURE. Thus far, it has been confirmed that PURE can be successfully applied to sputum, blood, serum, and swab samples. The mechanism of PURE is described below:

1. An aliquot of sample (blood, sputum, etc.) is added to the alkaline-based extraction solution and treated by heat to lyse the pathogens.

2. The sample solution is treated with adsorbent powder to remove inhibitory materials contained in samples and to neutralize the solution without any loss of target DNA.

3. After separating the solution from the powder by filtration, the obtained filtrate containing target DNA molecules can be used for reconstituting dried LAMP reagents, which are deposited to the lids of LAMP reaction tubes. 
A)

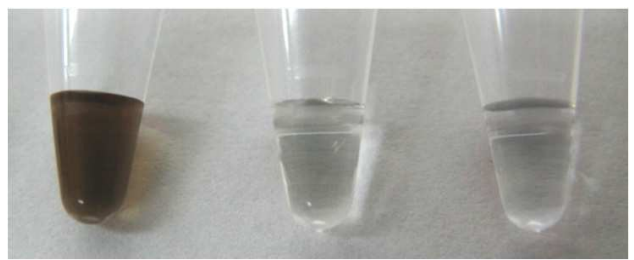

B)

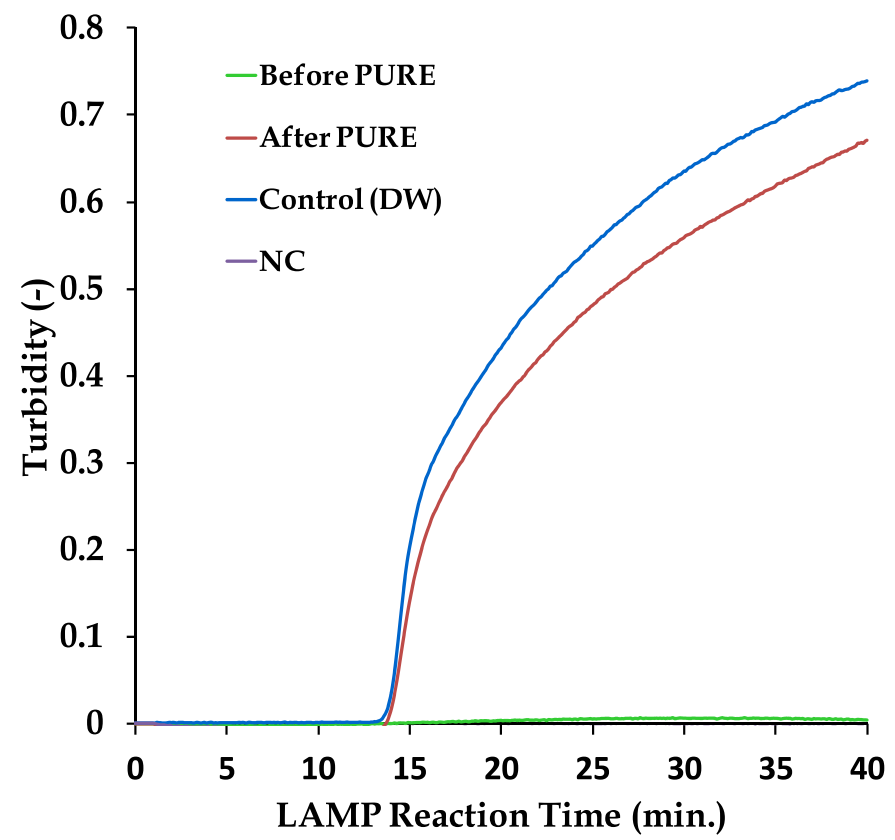

Fig. 3. Performance of the PURE-LAMP system applied for blood processing A) Pictures of the blood sample solution (3.6\% blood in an alkaline-based extraction solution) before and after PURE treatment. Left, Before PURE treatment; Centre, After PURE treatment; Right, distilled water (reference).

B) LAMP kinetics obtained by real-time turbidimetry for the 3 sample solutions with 1,000 copies of a template DNA spiked prior to PURE treatment.Green line, Before PURE treatment (directly added the solution to LAMP reaction); Red line, after PURE treatment; Blue line, control (distilled water); Purple line, negative control.

Figure 3 shows the performance of the PURE method applied for blood processing. An aliquot of blood was mixed with the extraction solution and heated at $70{ }^{\circ} \mathrm{C}$ for 5 minutes. Almost colorless solutions have been obtained by mixing the solution with the adsorbent powder (Fig. 3-A). The graph of real-time turbidimetry (Mori et al., 2004) in figure 3-B shows the LAMP kinetics for the 3 samples using 1,000 copies of a spiked template DNA. Untreated blood samples did not provide a positive reaction due to the inhibition from blood and extraction solution. However, PURE-treated blood samples showed almost the same kinetics as those of distilled water, indicating that PURE can remove inhibitory materials quite effectively from the blood samples without any loss of DNA. 
Figure 4 shows the overall process of the PURE-LAMP assay system. First, an aliquot of sample is placed in a heating tube and heated at an optimized temperature $\left(70-90^{\circ} \mathrm{C}\right)$ to lyse the target pathogen. Then, the heating tube is attached to an adsorbent tube, and the treated solution is vigorously mixed with adsorbent powder. Next, the injection cap is inserted into the absorbent tube, which is then squeezed to elute the solution containing purified DNA. These processes can be performed in approximately 10 minutes or less for a particular sample. The LAMP reagents deposited in the lid of the tube are reconstituted by shaking the tube several times and then incubating it at around $65^{\circ} \mathrm{C}$ for $30-40$ minutes. Finally, the results of amplification are detected by simply observing the fluorescence using the LED lights provided in the incubator. As mentioned above, we have successfully developed simple technologies for all the 3 steps required in molecular diagnostics, that is, the PURE method for sample preparation, the LAMP for amplification, and the calcein method for detection. Therefore, a combination of these technologies can be considered as a platform for a new molecular diagnostic tool with the desired simplicity.

\section{Application of the newly developed platform for diagnosing tropical diseases}

The developed platform has been applied to the following 3 tropical diseases to evaluate its performance as a practical diagnostic system.

\subsection{Malaria and human African trypanosomiasis}

Malaria is 1 of the 3 major infectious disease endemics in most tropical countries. More than 500 million people have been infected, and more than 1 million people die from malaria each year, mostly infants and pregnant women. Of the 4 malaria causing species, Plasmodium falciparum often causes severe, acute, and fatal malaria. In most developing countries, malaria is confirmed mainly by a blood smear test, although the sensitivity of the test is not sufficient to detect the parasites in patients with early-stage malaria.

Dried LAMP reagents using P. falciparum (Pf)-specific primers and pan genus (Pg) primers were developed in this study. The Pg LAMP primers were designed on the basis of the homogeneous sequence shared by all the 4 malaria species, thus providing the same primer specificity for all the 4 species. The Pf-specific LAMP primers were designed based on mutations between the Pf sequence and the other 3 sequences, making the primer specific only to P. falciparum. If both Pf and Pg LAMP assays give positive results, it can be interpreted that the patient is infected by $P$. falciparum. On the other hand, if only the $\mathrm{Pg}$ LAMP assay gives positive results, the patient can be diagnosed with malaria caused by 1 or more of the other 3 malarial parasites.

The sensitivity of PURE and malaria-LAMP have been evaluated by using of cultured $P$. falciparum parasites obtained from the American Type Culture Collection (ATCC). As shown in figure 5, both Pf and Pg malaria-LAMP assays can detect down to 1 parasite in $1 \mu$ blood, processed by the PURE method. This sensitivity of 1 parasite/ $\mu$ l blood is much higher than that of smear microscopy test ( $\sim 50$ parasites/ $\mu$ l blood for routine tests in an endemic area (Moody, 2002)). It has been reported that the initial malarial symptoms appear after the accumulation of approximately 1,000 parasites/ml of blood (Andrews et al., 2005). Therefore, PURE-malaria-LAMP is sensitive enough to detect parasites in patients who present with the initial symptoms of malaria.

Human African trypanosomiasis (HAT) is one of the most neglected disease endemics in central African countries (Hotez, 2007). HAT is caused by an infection of protozoa 
Trypanosoma brucei gambiense or Trypanosoma brucei rhodesiense transmitted by tse-tse flies. The symptoms of HAT occur in 2 phases: hemolymphatic phase, followed by the neurological phase. If left untreated, the hemolymphatic phase permits the parasites to invade the central nervous system of the patient, resulting in fatal neurological symptoms such as coma and eventually death. Because no vaccine or preventative drug for HAT is available, and therapeutic drugs for HAT patients in the neurological phase causes severe side effects, a simple and sensitive diagnostic method is in high demand.

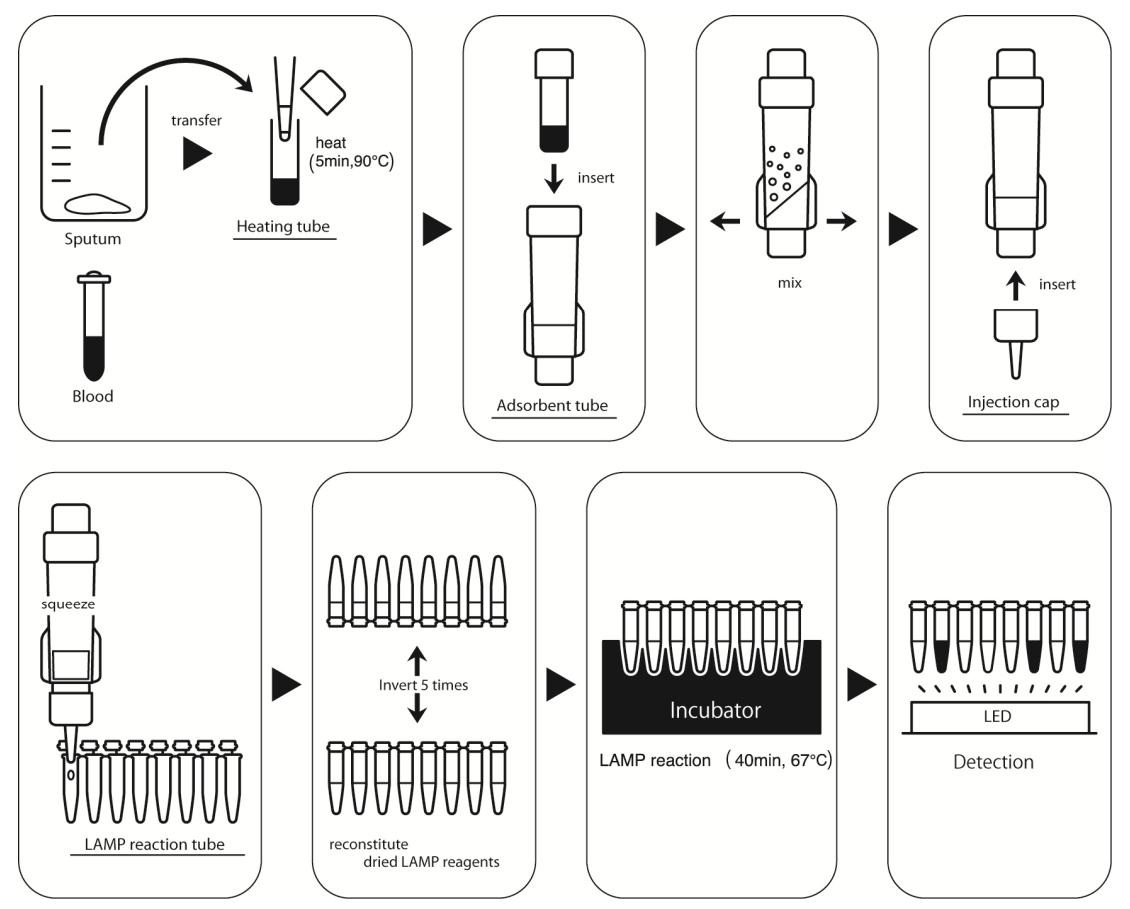

Fig. 4. Diagram of the procedures involved in the PURE-LAMP system

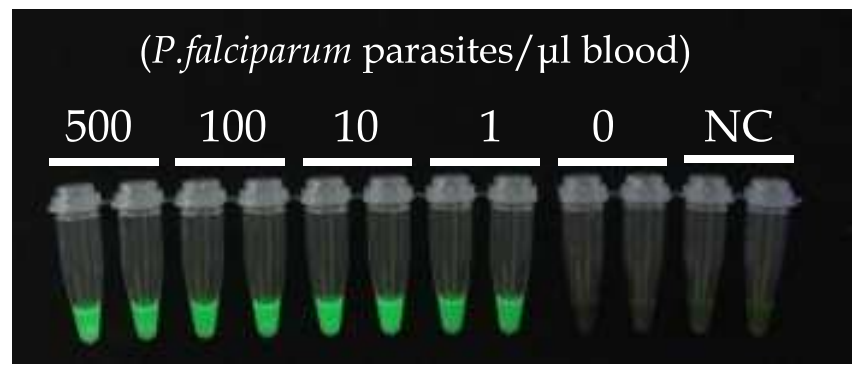

Fig. 5. Sensitivity of PURE-Malaria-LAMP

Thirty-five microliters of control blood spiked with cultured parasites ( $P$. falciparum, from ATCC) was treated with PURE and tested by $P$. falciparum-specific and Pan-genus LAMP. Left and right tubes contain the same parasite numbers and are those of Pf-LAMP and Pg-LAMP, respectively. 
To enhance sensitivity, the LAMP primers for HAT are designed on a multi-copy gene named RIME, which contains 80-250 copies in a parasite genome (Njiru et al., 2008). The efficacy of the PURE-HAT-LAMP assay was also evaluated by using uninfected control blood spike with cultured parasites. Positive results have been successfully obtained from samples with a parasite density of $100 / \mathrm{ml}$, indicating that the overall analytical sensitivity of PURE-HAT-LAMP can be estimated to be approximately 100 parasites $/ \mathrm{ml}$ blood. The sensitivities of the currently available smear microscopy tests have been reported to be between 100 and 10,000 parasites/ml blood (Chappuis., 2005). The sensitivity of PUREHAT-LAMP has been found to be over 10 times higher than that of the simple Giemsastained smear microscopy test, which is one of the most common diagnostic tests in HAT endemic areas, and is almost comparable with that of the mini-anion-exchange centrifugation technique, which is quite tedious and time consuming.

\subsection{Tuberculosis}

Tuberculosis (TB) is one of the most threatening airborne diseases worldwide. One-third of the world's population is thought to be infected with Mycobacterium tuberculosis and new infections occur at a rate of about 1 /second. Since the TB patients are concentrated in developing counties, including many tropical countries, TB is considered as a major poverty-related disease (Walker et al., 2003).

The sputum direct smear microscopy test is the only diagnostic method available for the detection of TB at peripheral laboratories in developing countries (Keeler et al., 2006). Because of the low sensitivity of the smear test, few patients with a low number of infected $\mathrm{TB}$ cells are often misdiagnosed as negative, thus preventing eradication of $\mathrm{TB}$. In order to overcome this situation, we attempted to apply the novel platform PURE-LAMP system for the diagnosis of TB.

As summarized in Table 1, PURE-TB-LAMP provided positive results for both smear- and culture-positive sputum samples collected from patients suspected with TB. Furthermore,

\begin{tabular}{ccccc}
\hline Direct Smear & Culture(Ogawa) & $\begin{array}{c}\text { PURE-TB-LAMP } \\
\text { (Positive/Total) }\end{array}$ & \% positive \\
\hline \multirow{2}{*}{ Positive } & Positive & $34 / 34$ & 100 \\
\hline \multirow{2}{*}{ Negative } & Positive & $15 / 25$ & 60 \\
\cline { 2 - 5 } & Colony & $100-200$ & $7 / 7$ & 100 \\
\cline { 3 - 5 } & Counts & $20-99$ & $6 / 8$ & 75 \\
\hline Negative & & $1-19$ & $2 / 10$ & 20 \\
\hline
\end{tabular}

Table 1. Summary of clinical performance of PURE-TB-LAMP

Sixty microliters of sputa obtained from patients suspected with TB in the Pham Ngoc Thach Hospital (PNTH; Vietnam) were analyzed by PURE-TB-LAMP. The performance of PURE-TB-LAMP was compared to those of the direct smear and culture method (Ogawa media) in terms of the positive ratios. Smear and culture tests for each sample were conducted according to the standardized protocols in PNTH. 
the PURE-TB-LAMP assay can detect the pathogen with $60 \%$ accuracy in smear-negative but culture-positive samples, and with $75 \%$ or more accuracy if 20 colonies are detected by the culture test using Ogawa media. This data clearly shows that the PURE-TB-LAMP method is reliable enough to be applied to the targeted peripheral smear centers in developing countries as an alternative method for the direct smear test.

\section{Conclusions}

As mentioned above, PURE and LAMP system have been newly developed and successfully applied as a novel diagnostic platform for the detection of infectious diseases, which are wildly endemic in the developing world. This platform has the advantage of being simple enough to be applicable in resource-limited facilities and its performances is higher than those of the existing diagnostic methods routinely employed in rural laboratories of most of the developing countries. Recently, a novel idea for performing LAMP without electricity has been proposed (LaBarre et al., 2011). Combination of that technology with the platform mentioned in this chapter would make it possible to realize the use of molecular diagnostics in poorer settings or even in field conditions.

Since the geographical distribution of malaria, HAT, and TB overlap in many of tropical countries (Cook \& Zumla, 2003), diagnostic tests for these diseases are often performed at the same rural laboratory in developing countries. The developed platform described in this study is a very useful tool in such laboratories because all the above mentioned diseases can be diagnosed using almost the same technique and the same simple incubator. This new technology can be beneficial as it reduces the initial costs associated with installing new equipments and preparing trained technicians for each target. This platform is potentially applicable to other pathogens, including those causing other neglected diseases such as leishmaniasis and Chagas' disease. The application of this platform could be extended to other diseases that threaten the heath and quality of life of patients in many tropical countries. This can also contribute to distribute them at more affordable rates because of the effect of mass production.

This platform can be considered as a gene point-of-care testing (g-POCT) device, which can also be used in developed countries. In fact, TB-LAMP has been approved as clinical in vitro diagnostics (IVD) in Japan and used along with PURE as a simple and fast screening test for patients suspected with TB. Since NALC-NaOH treatment for sputum is not necessary for PURE-TB-LAMP, turn-around-time of PURE-TB-LAMP is less than that of the decontaminated smear test, which is commonly adopted as the standard screening test for TB in most developed countries. Furthermore, LAMP reagents using similar concepts have been developed for the detection of the influenza virus (Nakauchi, 2011). We hope that the platform will contribute to the improvement of global health and benefit all those under the threat of infectious diseases.

\section{Acknowledgments}

We sincerely thank Dr. Satoshi Mitarai from the Research Institute of Tuberculosis in Japan, Dr. Noboru Inoue from Obihiro University of Agriculture and Veterinary Medicine in Japan, Dr. C.J. Sutherland and Dr. S.D. Polley from the London School of Hygiene and Tropical Medicine in the UK, and Dr. N.T. Lan from the Pham Ngoc Thach Hospital in Vietnam for their valuable help. This research was supported by the Foundation for Innovative New Diagnostics (FIND) in Switzerland. 


\section{References}

Andrews L, Andersen RF, Webster D, Dunachie S, Walther RM, Bejon P, Hunt-Cooke A, Bergson G, Sanderson F, Hill AV, Gilbert SC. (Jul 2005). Quantitative real-time polymerase chain reaction for malaria diagnosis and its use in malaria vaccine clinical trials. Am J Trop Med Hyg. Vol.73, No.1, pp.191-8.

Aziah I, Ravichandran M, Ismail A. (Dec 2007). Amplification of ST50 gene using dryreagent-based polymerase chain reaction for the detection of Salmonella typhi. Diagn. Microbiol. Infect. Dis. Vol.59, No.4, pp. 373-7.

Bendall K.E., Setzke E., Quandt A. (2002). Purification of Viral Nuculeic Acid, In: Nuculeic acids isolation methods, Bowien B. \& Dürre P. (Eds.) pp.53-59, American Scientific Publishers, ISBN 1-58883-018-7 2002

Chappuis F, Loutan L, Simarro P, Lejon V, Büscher P. (Jan 2005). Options for field diagnosis of human african trypanosomiasis, Clin. Microbiol. Rev. Vol.18, No.1, pp. 133-46.

Cook G.C. \& Zumla A. (Eds.) Manson's Tropical Diseases Twenty-first edition Elsevier Limited, ISBN 0-7020-2640-9

Goto M, Honda E, Ogura A, Nomoto A, Hanaki K. (Mar 2009). Colorimetric detection of loop-mediated isothermal amplification reaction by using hydroxy naphthol blue. Biotechniques. Vol.46, No.3, pp. 167-72.

Hotez PJ, Molyneux DH, Fenwick A, Kumaresan J, Sachs SE, Sachs JD, Savioli L. (Sep 2007)Control of neglected tropical diseases. N.Engl. J. Med. Vol.357, No.10, pp. 1018-27.

Iwamoto T, Sonobe T, Hayashi K. (Jun 2003). Loop-mediated isothermal amplification for direct detection of Mycobacterium tuberculosis complex, M. avium, and M. intracellulare in sputum samples., J.Clin. Microbiol. Vol. 41, No.6, pp. 2616-22

Jorgensen P, Chanthap L, Rebueno A, Tsuyuoka R, Bell D. (May 2006). Malaria rapid diagnostic tests in tropical climates: the need for a cool chain. Am J Trop Med Hyg. Vol.74, No.5, pp. 750-4.

Keeler E, Perkins MD, Small P, Hanson C, Reed S, Cunningham J, Aledort JE, Hillborne L, Rafael ME, Girosi F, Dye C. (Nov 2006). Reducing the global burden of tuberculosis: the contribution of improved diagnostics. Nature. 23;444 Suppl 1 pp. 49-57.

Kubo T, Agoh M, Mai le Q, Fukushima K, Nishimura H, Yamaguchi A, Hirano M, Yoshikawa A, Hasebe F, Kohno S, Morita K. (2010) Development of a reverse transcription-loop-mediated isothermal amplification assay for detection of pandemic (H1N1) 2009 virus as a novel molecular method for diagnosis of pandemic influenza in resource-limited settings. ., J Clin Microbiol. Vol.48, No. 3, pp. 728-35.

LaBarre P., Hawkins K.R., Gerlach J., Wilmoth J., Beddoe A., Singleton J., Boyle D., and Weigl B. (2011). A Simple, Inexpensive Device for Nucleic Acid Amplification without Electricity-Toward Instrument-Free Molecular Diagnostics in LowResource Settings, PLoS One. Vol. 6, No.5, e19738.

Moody A., (Jan 2002). Rapid Diagnostic Tests for Malaria Parasites, Clinical Microbiology Reviews. Vol.15, No.1, pp. 66-78

Mori Y, Hirano T, Notomi T. (Jan 2006). Sequence specific visual detection of LAMP reactions by addition of cationic polymers. BMC Biotechnol. Vol.10 6:3.

Mori Y., Kitao M., Tomita N., Notomi T., (2004). Real-time turbidimetry of LAMP reaction for quantifying template DNA, J Biochem Biophys Methods., Vol.59, No.2, pp. 145-57. 
Mori Y. \& Notomi T. (2009). Loop-mediated isothermal amplification (LAMP): a rapid. Accurate, and cost-effective diagnositic method for infectious diseases, J.Infect Chemother., Vol.15, No.2, pp.62-69.

Nagamine K., Hase T, Notomi T. (Jun 2002).Accelerated reaction by loop-mediated isothermal amplification using loop primers. Mol Cell Probes. Vol.16, No.3, pp. 223-9.

Nakauchi M, Yoshikawa T, Nakai H, Sugata K, Yoshikawa A, Asano Y, Ihira M, Tashiro M, Kageyama T. (Jan 2011). Evaluation of reverse transcription loop-mediated isothermal amplification assays for rapid diagnosis of pandemic influenza A/H1N1 2009 virus. J Med Virol. Vol.83, No.1, pp. 10-5.

Notomi T Okayama H., Masubuchi H., Yonekawa T., Watanabe K., Amino N. \& Hase T. (2000). Loop-mediated isothermal amplification of DNA, Nucleic Acids Res. Vol.28, No. 12, e63.

Njiru ZK, Mikosza AS, Matovu E, Enyaru JC, Ouma JO, Kibona SN, Thompson RC, Ndung'u JM. (Apr 2008). African trypanosomiasis: sensitive and rapid detection of the sub-genus Trypanozoon by loop-mediated isothermal amplification (LAMP) of parasite DNA. Int J Parasitol. Vol.38, No.5, pp. 589-99.

Parida M, Sannarangaiah S, Dash PK, Rao PV, Morita K. (Nov-Dec 2008). Loop mediated isothermal amplification (LAMP): a new generation of innovative gene amplification technique; perspectives in clinical diagnosis of infectious diseases. Rev Med Virol. Vol.18, No.6, pp. 407-21.

Polley SD, Mori Y, Watson J, Perkins MD, González IJ, Notomi T, Chiodini PL, Sutherland CJ. (Aug 2010). Mitochondrial DNA targets increase sensitivity of malaria detection using loop-mediated isothermal amplification. J Clin Microbiol. Vol.48, No.8., pp. 2866-7.

Rodrigues Ribeiro Teles FS, Pires de Távora Tavira LA, Pina da Fonseca LJ. (May-Jun 2010) Biosensors as rapid diagnostic tests for tropical diseases. Crit Rev Clin Lab Sci. Vol.47, No.3, pp. 139-69.

Tao ZY, Zhou HY, Xia H, Xu S, Zhu HW, Culleton RL, Han ET, Lu F, Fang Q, Gu YP, Liu YB, Zhu GD, Wang WM, Li JL, Cao J, Gao Q. (Jun 2011) Adaptation of a visualized loop-mediated isothermal amplification technique for field detection of Plasmodium vivax infection. Parasit Vectors. Vol.4, No.1, pp. 115.

Tomita N, Mori Y, Kanda H, Notomi T. (2008). Loop-mediated isothermal amplification (LAMP) of gene sequences and simple visual detection of products. Nat Protoc. Vol.3, No.5, pp. 877-82.

Walker D, Stevens W. (2003). The economics of TB control in developing countries. Expert Opin Pharmacother. Vol.4, No.3, pp. 359-68. 


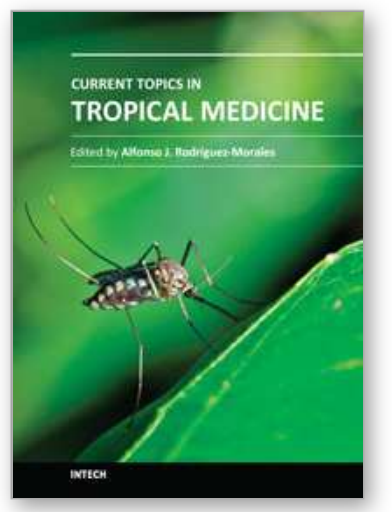

\author{
Current Topics in Tropical Medicine \\ Edited by Dr. Alfonso Rodriguez-Morales
}

ISBN 978-953-51-0274-8

Hard cover, 564 pages

Publisher InTech

Published online 16, March, 2012

Published in print edition March, 2012

Tropical Medicine has emerged and remained as an important discipline for the study of diseases endemic in the tropic, particularly those of infectious etiology. Emergence and reemergence of many tropical pathologies have recently aroused the interest of many fields of the study of tropical medicine, even including new infectious agents. Then evidence-based information in the field and regular updates are necessary. Current Topics in Tropical Medicine presents an updated information on multiple diseases and conditions of interest in the field. It Includes pathologies caused by bacteria, viruses and parasites, protozoans and helminths, as well as tropical non-infectious conditions. Many of them are considering not only epidemiological aspects, but also diagnostic, therapeutical, preventive, social, genetic, bioinformatic and molecular ones. With participation of authors from various countries, many from proper endemic areas, this book has a wide geographical perspective. Finally, all of these characteristics, make an excellent update on many aspects of tropical medicine in the world.

\title{
How to reference
}

In order to correctly reference this scholarly work, feel free to copy and paste the following:

Yasuyoshi Mori, Norihiro Tomita, Hidetoshi Kanda and Tsugunori Notomi (2012). Novel Molecular Diagnostic Platform for Tropical Infectious Diseases, Current Topics in Tropical Medicine, Dr. Alfonso Rodriguez-Morales (Ed.), ISBN: 978-953-51-0274-8, InTech, Available from: http://www.intechopen.com/books/current-topics-intropical-medicine/novel-molecular-diagnostic-platform-for-tropical-infectious-diseases

\section{INTECH}

open science | open minds

\section{InTech Europe}

University Campus STeP Ri

Slavka Krautzeka 83/A

51000 Rijeka, Croatia

Phone: +385 (51) 770447

Fax: +385 (51) 686166

www.intechopen.com

\section{InTech China}

Unit 405, Office Block, Hotel Equatorial Shanghai

No.65, Yan An Road (West), Shanghai, 200040, China

中国上海市延安西路65号上海国际贵都大饭店办公楼 405 单元

Phone: +86-21-62489820

Fax: $+86-21-62489821$ 
(C) 2012 The Author(s). Licensee IntechOpen. This is an open access article distributed under the terms of the Creative Commons Attribution 3.0 License, which permits unrestricted use, distribution, and reproduction in any medium, provided the original work is properly cited. 